\title{
Periodic Rosuvastatin or Atorvastatin Dosing Arrays (PRADA): Patient-Centered Practice
}

\author{
Theodore Christou • Hesham R. Omar • \\ Roger Dimitrov
}

Published online: 27 August 2014

(c) The Author(s) 2014. This article is published with open access at Springerlink.com

\begin{abstract}
Background Non-adherence is a major obstacle with long-term daily statin therapy.

Objective This retrospective study reviewed the medical records of patients with hyperlipidemia during an 8-year period in a private internal medicine practice. Periodic dosing was negotiated following several patients' refusal of statin therapy because of muscle aches or cost.

Methods The clinical impetus was patient adherence to statin therapy. Treatment was initiated by dispensing rosuvastatin or atorvastatin in a stepwise patient-directed approach (from two times/week to three times/week to every other day, up to five times/week). The primary endpoint was to assess the concentration of low-density lipoprotein cholesterol (LDL-C) and the total cholesterol/ high-density lipoprotein cholesterol (TC/HDL-C) ratio with patient-directed dosing intervals. The secondary endpoint was a head-to-head comparison of atorvastatin and rosuvastatin to evaluate the mean decrease in the LDL$\mathrm{C}$ and TC/HDL-C ratio.
\end{abstract}

\footnotetext{
T. Christou $(\varangle) \cdot$ H. R. Omar

Internal Medicine Department, Mercy Hospital and Medical Center, 2525 South Michigan Avenue, Chicago, IL 60616, USA

e-mail: tchristo@uic.edu

H. R. Omar

e-mail: hesham_engy2008@yahoo.com

R. Dimitrov

Biostatistics Department, Mercy Hospital and Medical Center,

Chicago, IL, USA

e-mail: rdimitrov@mercy-chicago.org
}

Results Chart review identified 46 patients who had been treated. Two patients with persistent myalgia terminated treatment before 12 weeks. Among the remaining 44 patients, 20 received doses of rosuvastatin from 15 to $100 \mathrm{mg}$ per week, and 24 received atorvastatin from 20 to $140 \mathrm{mg}$ per week. There was a significant decrease from pre-treatment in the mean TC/HDL-C ratio of 1.72 $(31.1 \%, P<0.0001)$ and mean LDL-C of $43.3 \mathrm{mg} / \mathrm{dL}$ (30.2\%, $P<0.0001)$. An independent samples $t$-test showed a non-significant reduction of the mean TC/HDL-C ratio and LDL-C with rosuvastatin versus atorvastatin.

Conclusion Periodic dosing of rosuvastatin or atorvastatin using a gradual, patient-directed, stepwise approach guided by cholesterol levels is an effective method of lipid lowering and carried a favorable $95.6 \%$ adherence rate.

\section{Introduction}

Lowering the low-density lipoprotein (LDL-C) and total cholesterol/high-density lipoprotein cholesterol (TC/HDLC) [1] ratio is associated with significant reduction in coronary atherosclerotic morbidity and mortality rates $[2$, $3]$. Studies have found myalgia and muscle cramps reported by $10.5-60 \%$ of patients treated with statins $[4,5]$. In clinical practice, patient concerns over cost and adverse effects must be addressed and at times negotiated to achieve a therapeutic goal. During any 2-year period, between 25.4 and $40.1 \%$ of patients may become nonadherent to a daily statin regimen [6]. Periodic dosing of rosuvastatin or atorvastatin has been described in previous studies [7-9] given their long physiologic half-life and a plasma half-life seven times greater than simvastatin. We examine the process of periodic dosing of rosuvastatin or 
atorvastatin to reach therapeutic goals and promote patient adherence over an 8-year period.

\section{Methods}

In 2002, several patients in a private internal medicine practice, who had failed to improve their lipid profile with non-pharmacologic options, had stopped simvastatin treatment because of myalgias. These patients were given the option to try periodic statin therapy to achieve a TC/ HDL-C ratio less than 5. Over the next 6 months, a selection process was standardized and offered to other patients who stopped taking their statin because of myalgias or cost. Patients who were adherent to their prescribed statin treatment were excluded. During a 7-month review of medication profiles, 46 patients (Table 1) were identified who had chosen a non-daily dosing schedule during an 8 -year period since 2002. Each patient was given 14 tablets: 20 patients were given rosuvastatin $5 \mathrm{mg}$ tablets, 24 were given $10 \mathrm{mg}$ tablets of atorvastatin, and 2 patients were unable to tolerate any dose. Instructions for the first week were to take one tablet on Monday and a second tablet on Wednesday. They were then to take a tablet on Monday, Wednesday, and Friday for the next 4 weeks, and follow-up in the office with a lipid profile. During the office visit, post-treatment activity and lifestyle concerns were addressed, as well as the results of the lipid profile. Following a discussion with the patient about the lipid profile results and their perceptions of either the $30 \mathrm{mg}$ weekly dose of atorvastatin or $15 \mathrm{mg}$ of rosuvastatin; each patient was given the choice of maintaining the therapy, doubling the $\mathrm{mg}$ dose, or increasing the frequency up to 5 days per week. The initial post-treatment interview and lipid profile directed that a prescription should be given for 30 additional tablets of the negotiated dose to "take as directed." Subsequent lipid testing was performed at 3- to 6-month

Table 1 Patient characteristics

\begin{tabular}{llll}
\hline & $\begin{array}{l}\text { All } \\
(N=44)\end{array}$ & $\begin{array}{l}\text { Atorvastatin } \\
(N=24)\end{array}$ & $\begin{array}{l}\text { Rosuvastatin } \\
(N=20)\end{array}$ \\
\hline Age, years & $69(10.6)$ & $71(10.3)$ & $66(10.6)$ \\
Female (\%) & 31.8 & 50.0 & 50.0 \\
Male (\%) & 68.2 & 56.7 & 43.3 \\
Pre-treatment LDL & $143(38.5)$ & $138(43)$ & $149(32)$ \\
Pre-treatment CH/HDL & $5.4(1.3)$ & $5.2(1.3)$ & $5.6(1.3)$ \\
Doses per month & $14.9(3.5)$ & $14.7(3.6)$ & $15.1(3.6)$ \\
Months of treatment & 36.9 & 36.5 & 37.3 \\
\hline
\end{tabular}

Data are presented as mean (SD) unless otherwise indicated $C H$ cholesterol, $H D L$ high-density lipoprotein, $L D L$ low-density lipoprotein, $S D$ standard deviation intervals until the TC/HDL-C goal of less than 5 was achieved. Stepwise dosing was titrated down if myalgias arose or as per patient request. The primary endpoint in a retrospective chart review was to assess the concentration of LDL-C and the TC/HDL-C ratio with patient-selected atorvastatin and rosuvastatin dosing intervals. The secondary endpoint was a head-to-head comparison of the lipid reductions with atorvastatin versus rosuvastatin. Statistical analyses to compare pre-treatment and post-treatment LDL-C level and TC/HDL-C ratio in the whole group were performed using a paired-samples $t$-test. An independent samples $t$-test was conducted to compare pre- and post-treatment LDL-C level and TC/HDL-C ratio reduction in the rosuvastatin verses atorvastatin groups. Results were expressed as means and $95 \%$ confidence intervals (CIs). A $P$ value of less than 0.05 was considered statistically significant. All data were processed and analyzed using SPSS, version 21 (IBM Inc., Armonk, NY, USA). An institutional review board approved the ethics of this study.

\section{Results}

The patients ranged from 46 to 79 years of age and were treated for a mean duration of 37 months (range 2-99) with titration of atorvastatin from 10 to $40 \mathrm{mg}$ and rosuvastatin from 5 to $20 \mathrm{mg}$. Two patients $(4.3 \%)$ failed to tolerate any dose of statin, and three patients $(6.5 \%)$ decided to take their medication no more than twice weekly (which was not related to myalgias). Of the 44 patients treated with either rosuvastatin or atorvastatin, there was a statistically significant decrease from baseline in the mean LDLC level of $43.3 \mathrm{mg} / \mathrm{dL} \quad(30.2 \%)$ (95 \% CI 34-52.6, $P<0.0001$ ) (Fig. 1, left). In the atorvastatin group, the target TC/HDL-C ratio was achieved in two patients $(8.3 \%)$ with 2-days/week therapy, in eight patients $(33.3 \%)$ with 3-days/week therapy, in ten patients $(41.7 \%)$ with therapy every other day, and in four patients $(16.7 \%)$ with 5-days/week therapy. In the rosuvastatin group, the target TC/HDL-C ratio was achieved in one patient $(5 \%)$ with 2-days/week therapy, in eight patients (40\%) with 3-days/week therapy, in seven patients (35\%) with therapy every other day, and in four patients $(20 \%)$ with 5-days/week therapy. There was also a statistically significant decrease from pre-treatment levels in the mean TC/HDL-C ratio of $1.72(31.1 \%)$ (95\% CI 1.4-2, $P<0.0001$ ) (Fig. 1, right). In terms of total weekly dose of these two statins, $50 \%$ of the patients were controlled with 17.5-30 mg per week of rosuvastatin or with 20-50 mg per week of atorvastatin (Fig. 2). The maximum weekly dose of $100 \mathrm{mg}$ or more was needed in $5 \%$ of the rosuvastatin group and in $12.5 \%$ of the atorvastatin group. 

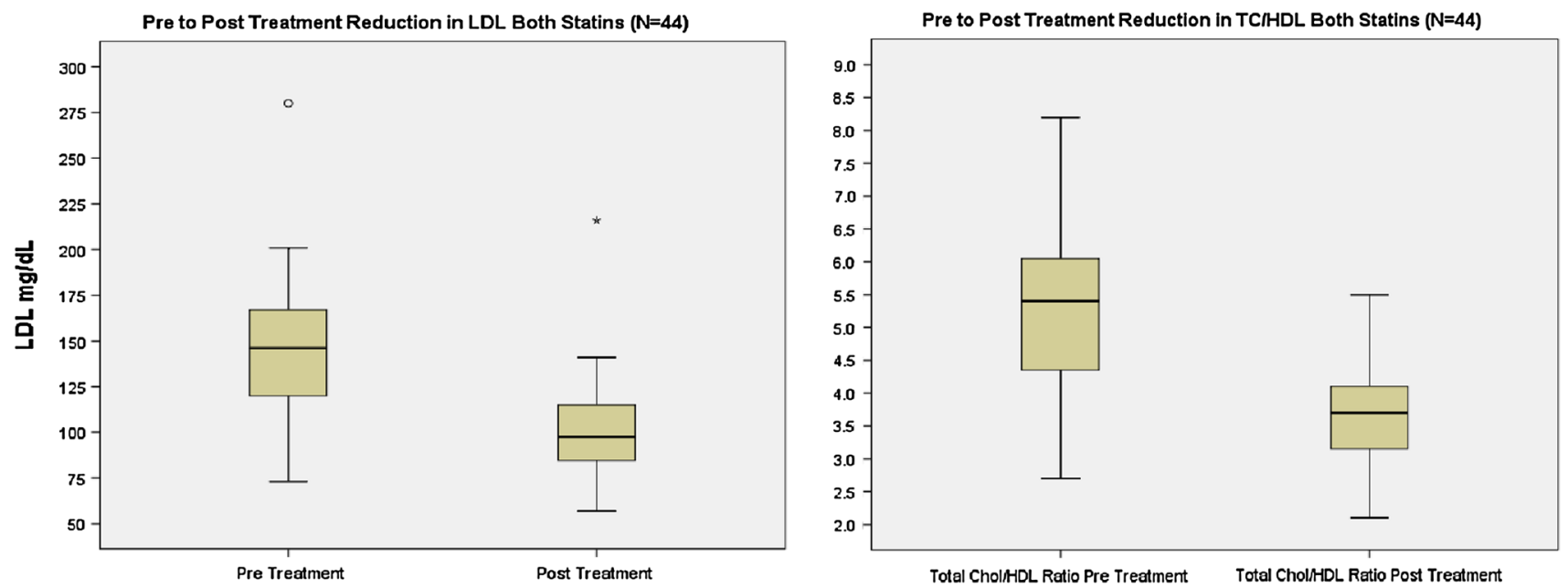

Fig. 1 Comparison of pre- and post-treatment LDL-C level (left) and TC /HDL ratio (right) in the 44 patients. HDL high-density lipoprotein, $L D L-C$ low-density lipoprotein cholesterol, $T C$ total cholesterol

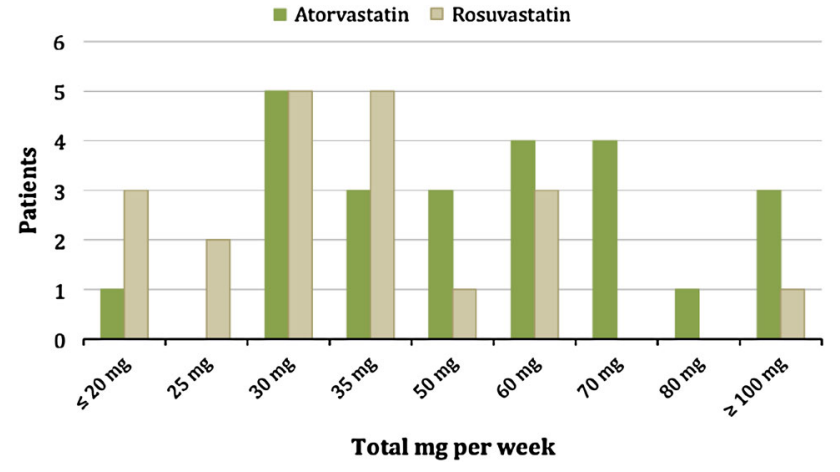

Fig. 2 Weekly cumulative dose of rosuvastatin or atorvastatin
The head-to-head comparison failed to show a statistically significant difference between rosuvastatin and atorvastatin in the reduction in mean LDL-C concentration. Rosuvastatin produced a $33.9 \%$ reduction compared with a $26.8 \%$ reduction with atorvastatin, with a mean difference in absolute LDL-C reduction of $13.62 \mathrm{mg} / \mathrm{dL}$ between groups (95\% CI $-4.8-32, P=0.143$ ) (Fig. 3, left). Reduction of the mean TC/HDL-C ratio with rosuvastatin was $32.9 \%$ compared with a $30.8 \%$ reduction with atorvastatin. The mean difference in TC/HDL-C ratio reduction of 0.27 between both groups was not statistically significant $95 \%$ CI $-0.9-0.4, P=0.399$ ) (Fig. 3, right).
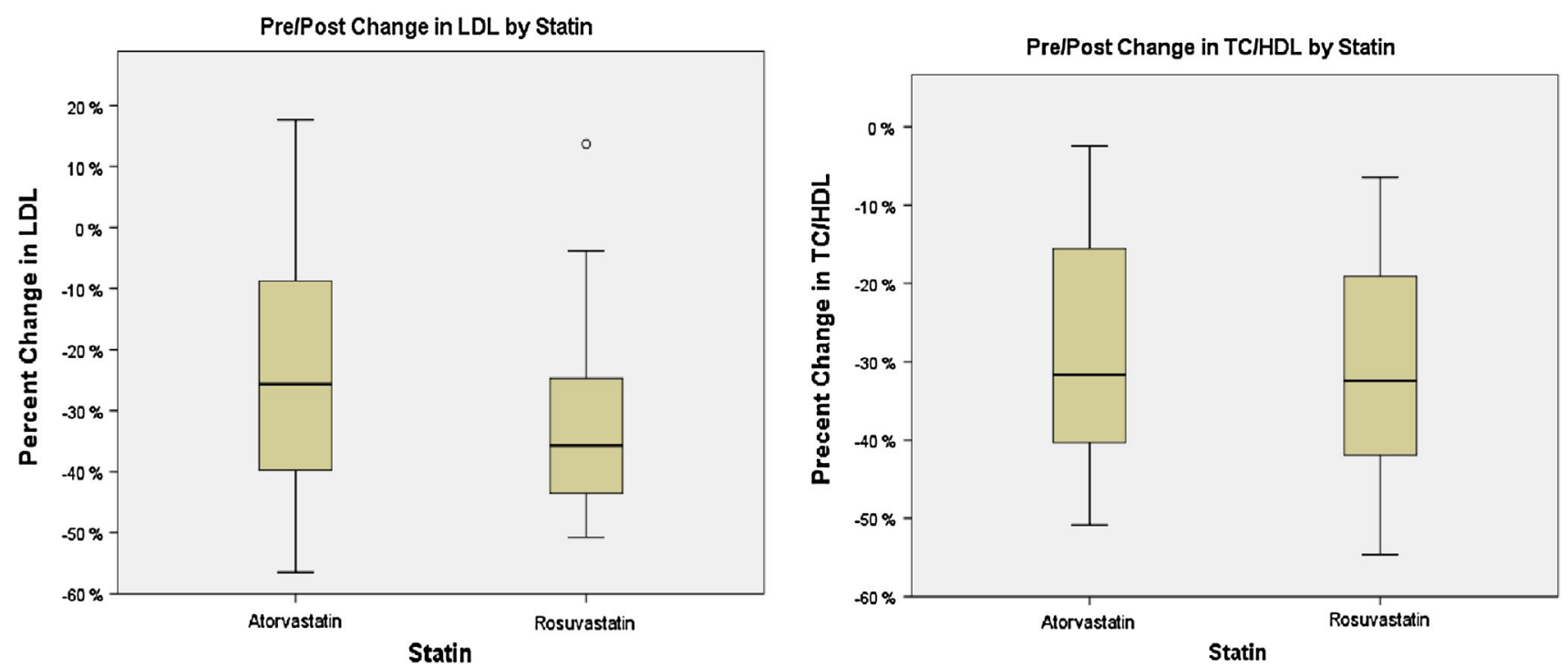

Fig. 3 Head-to-head comparison of LDL-C level (left) and TC/HDL ratio (right) reduction in the atorvastatin and rosuvastatin group. HDL highdensity lipoprotein, $L D L-C$ low-density lipoprotein cholesterol, $T C$ total cholesterol 


\section{Discussion}

The clinical impact of long-term statin use may be compromised by patient concerns, including myalgias and cost, resulting in decreased adherence. This study demonstrates that periodic dosing of rosuvastatin or atorvastatin is effective in achieving a desirable LDL-C and TC/HDL-C ratio for up to 8 years. Rosuvastatin and atorvastatin were selected because of their uniquely long in vivo activity. The half-life of atorvastatin is approximately $14 \mathrm{~h}$, and the half-life of its HMG-CoA reductase inhibiting metabolites approaches 20-30 h. Rosuvastatin has an extended half-life of $19 \mathrm{~h}$ as well as enterohepatic recirculation in most patients [10]. The slow hepatic metabolism of 5-50\% of the drug has been shown to occur over 3 days in in vitro studies [11]. Among the 44 patients who received either rosuvastatin or atorvastatin, there was no statistically significant difference between both drugs in lowering the LDL-C and TC/HDL-C ratio.

Although rosuvastatin will soon be generic, and atorvastatin has been available in a generic form for several years, when the study was initiated, the cost of rosuvastatin or atorvastatin was over \$US5 per tablet. During the course of this study, our group of patients saved approximately \$US42,195 with periodic treatment compared with conventional daily dosing. Using our data, the cost savings for treating 1 million patients, at the current cash cost of 90 generic atorvastatin for \$US53 [12] would be a savings of \$US197 million per year. Supported by the pharmacokinetics of these two drugs, a dosing schedule of every other day may permit effective treatment with a $50 \%$ savings to the patient. Periodic rosuvastatin and atorvastatin dosing from once weekly to every other day has been studied and been shown to result in significant cholesterol reduction. Our study is the first to adapt a pragmatic stepwise approach, offering patient input to manage their hyperlipidemia. During the 8-year period, the patients were given the opportunity to choose a dosage regimen based on how they responded to treatment with a defined goal of $\mathrm{TC} /$ HDL-C ratio $<5$. Using a patient-directed stepwise approach, we demonstrated sustained patient adherence of $95.7 \%$, which compares favorably with figures for daily dosing from the literature. Several studies have found 36-60\% of the patients were adherent to prescribed statin dosing after 12 months [13, 14]. Patient-directed therapy promoted an acceptable quality of life while reaching the stated lipid treatment goals in an office setting. This study adds evidence to the utility of a patient-centered approach to managing hyperlipidemia in select patients. Limitations of the study include the small cohort and the retrospective design nature. There was no cardiovascular endpoint measurement to see whether this treatment strategy was associated with favorable cardiovascular outcomes compared with daily statin dosing. Although no cardiac events occurred during the 8 years reviewed, additional comparative studies with a larger patient population are required to confirm the long-term cardioprotective effects of periodic statin dosing.

Conflicts of interest The authors have no conflicts of interest and have received no funding or financial support in the execution or preparation of this study.

Author participation Each of the authors participated in the data collection, organization, and writing of this manuscript. Mr. Dimitrov was the statistician who analyzed the data.

Open Access This article is distributed under the terms of the Creative Commons Attribution Noncommercial License which permits any noncommercial use, distribution, and reproduction in any medium, provided the original author(s) and the source are credited.

\section{References}

1. Lemieux I, Lamarche B, Couillard C, et al. Total cholesterol/ HDL cholesterol ratio vs LDL cholesterol/HDL cholesterol ratio as indices of ischemic heart disease risk in men: The Quebec Cardiovascular Study. Arch Intern Med. 2001;161(22):2685-92.

2. The Long-Term Intervention with Pravastatin in Ischemic Disease (LIPID) Study Group. Prevention of cardiovascular events and death with pravastatin in patients with coronary heart disease and a broad range of initial cholesterol levels. N Engl J Med. 1998;339(19):1349-57.

3. Heart Protection Study Collaborative Group. MRC/BHF Heart Protection Study of cholesterol lowering with simvastatin in 20,536 high-risk individuals: a randomized placebo-controlled trial. Lancet. 2002;360(9326):7-22.

4. Bruckert E, Hayem G, Dejager S, Yau C, Bégaud B. Mild to moderate muscular symptoms with high-dosage statin therapy in hyperlipidemic patients- the PRIMO study. Cardiovasc Drugs Ther. 2005;19(6):403-14.

5. Cohen JD, et al. Understating statin use in America and gaps in patient education (USAGE): an internet-based survey of 10,138 current and former statin users. J Clin Lipidol. 2012;6:208-15.

6. Jackevicius CA, Mamdani M, Tu JV. Adherence with statin therapy in elderly patients with and without acute coronary syndromes. JAMA. 2003;288:462-7.

7. Mackie BD, Satija S, Nell C, Miller J 3rd, Sperling LS. Monday, Wednesday, and Friday dosing of rosuvastatin in patients previously intolerant to statin therapy. Am J Cardiol. 2007;99(2):291.

8. Kennedy SP, Barnas GP, Schmidt MJ, Glisczinski MS, Paniagua AC. Efficacy and tolerability of once-weekly rosuvastatin in patients with previous statin intolerance. J Clin Lipidol. 2011;5:308-15.

9. Marcus FI, Baumgarten AJ, Fritz WL, Nolan PE. Alternate-day dosing with statins. Am J Med. 2013;126(2):99-104.

10. McCormick AD, Butters CJ, Miles GS, Baba T, Touchi A, Yamaguchi Y. ZD4522-An HMG-CoA reductase inhibitor free of metabolically mediated drug interactions: metabolic studies in human in vitro systems. J Clin Pharmacol. 2000;40:1055.

11. Martin PD, Warwick MJ, Dane AL, Hill SJ, Giles PB, Phillips PJ, Lenz E. Metabolism, exceretion, and pharmacokinetics of rosuvastatin in healthy adult male volunteers. Clin Ther. 2003;25(11):2822-35. 
12. Atorvastatin (Lipitor): prices for 30 tablets of atorvastatin $20 \mathrm{mg}$ (generic). http://www.goodrx.com/atorvastatin/price. Accessed July 2014

13. Benner JS, Glynn RJ, Mogun H, et al. Long-term persistence in use of statin therapy in elderly patients. JAMA. 2002;288: $455-61$.
14. Mantel-Teeuwisse AK, Goettsch WG, Klungel OH, De Boer A, Herings RMC. Long term persistence with statin treatment in daily medical practice. Heart. 2004;90:1065-6. 